\title{
The (110) and (320) surfaces of a Cantor alloy
}

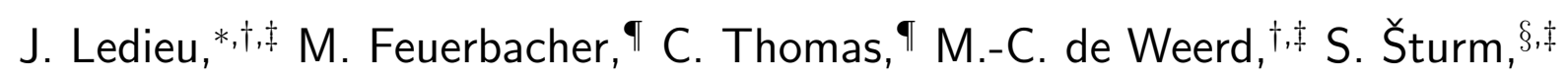
M. Podlogar,,$^{\S, \ddagger}$ J. Ghanbaja, ${ }^{\dagger, \ddagger}$ S. Migot, ${ }^{\dagger, \ddagger}$ M. Sicot, ${ }^{\dagger, \ddagger}$ and V. Fournée $e^{\dagger, \ddagger}$

$\dagger$ Université de Lorraine, CNRS, IJL, F-54000 Nancy, France

$\ddagger$ International Associated Laboratory PACS2, CNRS Université de Lorraine, Nancy, France and Jožef Stefan Institute, Ljubljana, Slovenia

\Ernst Ruska-Centre for Microscopy and Spectroscopy with Electrons and Peter Grünberg Institute, Forschungszentrum Jülich GmbH, 52425 Jülich, Germany §Jožef Stefan Institute, Jamova Cesta 39, 1000 Ljubljana, Slovenia

E-mail: julian.ledieu@univ-lorraine.fr 


\section{Graphical Abstract}

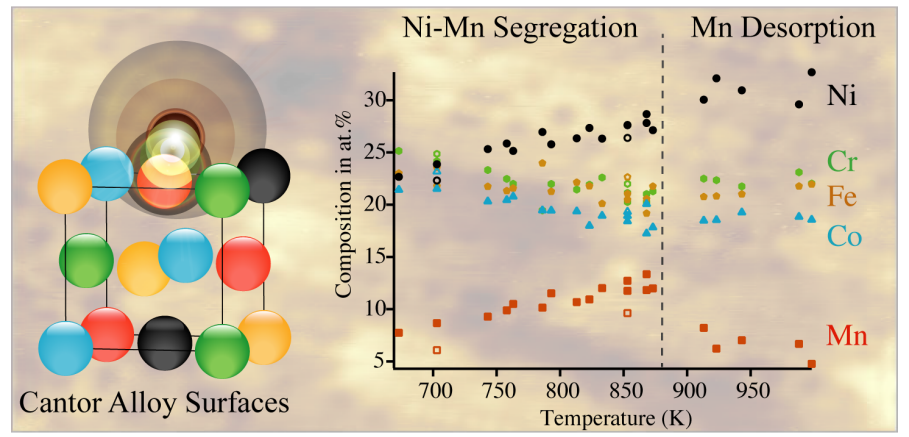

KEYWORDS: High entropy alloys, Surface, Surface segregation, STEM, XPS. 


\begin{abstract}
The (110) and (320) surfaces of the single-phase FeCrMnNiCo solid solution have been studied on two adjacent millimeter size grains using surface science and transmission electron microscopy (TEM) techniques. The structural and chemical evolutions of the high entropy alloy (HEA) surfaces have been determined for various sputtering conditions, annealing temperatures and durations. Up to $873 \mathrm{~K}$, angle-resolved x-ray photoelectron spectroscopy measurements indicate a clear Mn and Ni surface cosegregation. We propose that the surface segregation of Mn is driven by its low surface energy. The attractive interaction between $\mathrm{Mn}$ and Ni promotes Ni segregation which accompanied the Mn diffusion to the surface. Regarding the structures investigated by low energy electron diffraction and scanning tunneling microscopy, the (320) surface presents a terraced morphology with an ordered structure consistent with a $(1 \times 1)$ termination. On the contrary, the (110) surface reveals an important degree of structural disorder and local reconstructions. Its highly anisotropic morphology resembles rows propagating along the [001] direction. Above $873 \mathrm{~K}$, Mn desorption occurs while the Ni content keeps increasing linearly with the temperature. TEM analysis show no evidence for HEA decomposition into metallic or intermetallic phases even after repeated annealing and sputtering cycles. The above results set the upper temperature limit above which the surface stoichiometry departs from the quinary HEA concept. It also defines the temperature range for the use of FeCrMnNiCo based coating under high vacuum conditions and for aerospace applications.
\end{abstract}




\section{Introduction}

The term high entropy alloys (HEA), initially introduced by Yeh et al. ${ }^{1}$ refers to metallic random solid solution made of at least five elements each of which in an equi- or nearequiatomic concentration. With no principal element, these materials adopt simple crystallographic structures such as body centered cubic $(b c c)$, face centered cubic $(f c c)$ and more recently hexagonal close packed $(h c p)$ structure solid solution phases. ${ }^{2}$ The concept of HEA has offered new perspectives in material and metallic alloy design with a wealth of elemental combination ${ }^{3}$ and composition resulting in unique properties. It has already led to the identification of superconductivity in refractory $\mathrm{Ta}_{34} \mathrm{Nb}_{33} \mathrm{Hf}_{8} \mathrm{Zr}_{14} \mathrm{Ti}_{11} \mathrm{HEA}^{4}$ and more recently to magnetically soft transition metal HEAs in the FeCoNiPdCu system which performs comparatively to the best commercial soft magnets. ${ }^{5}$ In literature, the majority of the studies remains devoted to the characterisation of the mechanical properties depending on the microstructure and composition. Among the variety of alloys, several refractory-based HEAs appear as potential candidates for applications in transport or aerospace industries due to their high melting temperature combined with high hardness. ${ }^{6,7}$ Remarkable mechanical properties have also been highlighted for one of the most studied HEA namely the equiatomic single phase FeCrCoMnNi alloy also referred as Cantor alloy. ${ }^{8}$ Here, very high fracture toughness and tensile strengths which further improve at cryogenic temperature result in an excellent tolerance to damage. ${ }^{9}$ The use of HEA is also considered in the form of coating due to their excellent surface properties compared to conventional alloys including oxidation, corrosion and wear resistance. ${ }^{10-12}$ The deposition conditions will vary with the selected growth techniques (chemical, physical...) and HEA systems. To achieve the growth of a single phase HEA coating, several parameters including annealing treatment, adsorption sequences should be fined tuned. This is key to preserve the desired film properties which would be affected by its microstructure, the different interfaces in presence and possible surface effects.

Finally, quinary up to octonary HEA nanoparticles (NP) have been recently synthe- 
sized by carbon thermal shock method. ${ }^{13}$ With the concept of HEA preserved down to the nanoscale, the authors have demonstrated the catalytic properties of noble PtPdIrRhRu HEA-NP towards ammonia oxidation, hence adding to the long list of HEA attractive physical and chemical properties.

To further understand the potential of HEA towards heterogeneous catalysis or as functional coating, it is of paramount importance to determine the atomic and chemical ordering at HEA surfaces along with their evolution under different conditions (pressure, temperature for instance). To this end, two model surfaces will be investigated namely the FeCrMn$\mathrm{NiCo}(110)$ and (320). The latter can be regarded as a $11.3^{\circ}$ miscut from the low index (110) plane, the structural model of the surface consisting of two (110) and one (100) facets. The study on these HEA surfaces will bring answers to fundamental questions on surface effects. For instance, would a random solid-solution be preserved at the topmost surface? Is surface reconstruction expected on such systems lacking chemical ordering? Here, FeCrMnNiCo(320) and (110) surfaces have been characterised under ultra high vacuum (UHV) conditions following different consecutive preparations. With no report available on HEA single crystal surfaces, this study sets the temperature limits to expose the Cantor alloy in the form of a film or coating under UHV conditions, reveals an history effect on the HEA surface composition upon repeated annealing cycles, highlights $\mathrm{Mn} / \mathrm{Ni}$ co-segregation in the near-surface region and demonstrates a morphology difference between (320) and (110) surfaces.

\section{Experimental details}

The present study has been performed on a single phased sample grown by the Czochralski method using high purity elements. The equiatomic nominal composition of FeCrMnNiCo is not maintained after the growth. A depletion of about 8 at.\% Mn (due to its high vapour pressure $^{14}$ ) is measured by energy dispersive x-ray spectroscopy (EDS), while the homogeneous elemental distribution is preserved. The sample $\mathrm{Fe}_{23.8} \mathrm{Co}_{22.8} \mathrm{Cr}_{22.3} \mathrm{Mn}_{12.0} \mathrm{Ni}_{19.1}$ consists 
of four adjacent millimeter-size grains (see Fig.S1). The sample surface has been aligned using back reflection Laue x-ray diffraction and cut such that the largest grain presents a (110) surface orientation. This alignment has resulted in the surface of the second largest grain to be perpendicular to the [320] direction. In that geometry, the two other grains located only on the sample edges have a much smaller contribution to the total surface area. Prior to insertion under UHV, the sample surface has been polished down to $1 / 4 \mu m$ diamond paste. The surface is then mirror-like with no distinction of grain boundaries under polarized light. Once in UHV, the sample preparation consists of $\mathrm{Ar}^{+}$ion sputtering (1.5-2.5 keV) and annealing cycles and the grain boundaries reappear. The sample temperature has been monitored using an infrared optical pyrometer (emissivity set to 0.10) along with a K-type thermocouple attached to the heating stage. The scanning tunneling microscopy (STM), x-ray photoelectron spectroscopy (XPS) using a non-monochromatised $\mathrm{Mg} \mathrm{K} \alpha$ source, and low energy electron diffraction (LEED) measurements have been performed with the sample at $300 \mathrm{~K}$. The XPS measurements have been collected always for the same sample position at normal take-off angle (except for angle-resolved XPS measurements), hence probing the same surface area. Due to multiple overlapping between Auger and $2 p$ core level spectra, the XPS intensities have been extracted from the five element $3 p$ core levels using a Shirley background (see Fig.S2). The XPS measurements were also used to check the cleanliness of the surfaces. In a separate UHV platform equipped with scanning electron and Auger microscopy (SEM/SAM) techniques, the sample has been re-prepared using similar sputtering and annealing cycles and it has been possible to control the spatial chemical distribution at HEA surfaces. Moreover, SEM and EDS measurements have been carried out following these UHV experiments to analyse the overall surface topography (then exposed to air) and in particular the bulk composition of grains.

In a parallel set of experiments, the UHV prepared surfaces have been capped by a 10 monolayers thick Fe film deposited under UHV at $300 \mathrm{~K}$ before exposing the surface to air. Then, electron-transparent lamellae have been cut perpendicularly to the (320) and (110) 
surfaces by Focus Ion beam (FIB). Cross-sectional observations at the Fe/HEA interface, beneath this interface and in the bulk have been carried out using scanning transmission electron microscopy (STEM). The elemental distributions on the lamellae have been mapped using electron energy loss spectroscopy (EELS) and EDS measurements.

\section{Results}

\subsection{Surface composition}

Dealing with a five-element random solid solution, it is important to determine the evolution of the HEA surface stoichiometry as a function of the annealing time and temperatures. To this end, XPS measurements have been carried out after annealing the FeCrMnNiCo between $673 \mathrm{~K}-1000 \mathrm{~K}$ and for up to 4 hours. The composition has been also determined directly after sputtering the sample. The XPS measurements under high magnification mode (probing a small area region of about $1.5 \mathrm{~mm} \times 3 \mathrm{~mm}$ ) have been collected at the centre of the sample. Due to the sample configuration, the surface composition is measured over both the (110) and (320) grains.

First, angle-resolved XPS measurements have been carried out after sputtering the sample. The concentration of each element remains constant between $0^{\circ}$ and $70^{\circ}$ photoelectron take-off angle, hence leading to the conclusion that there is no preferential elemental sputtering (see Fig.S3). The surface stoichiometry measured at $\mathrm{Fe}_{21.7} \mathrm{Co}_{22.5} \mathrm{Cr}_{25.1} \mathrm{Mn}_{7.8} \mathrm{Ni}_{22.9}$ is comparable to the composition obtained after annealing the sample to $673 \mathrm{~K}$ (see Fig.1(a)), temperature at which a LEED pattern is already observed. With the uncertainty inherent to the XPS technique, the above composition should be considered as our nominal FeCrMnNiCo composition.

Next, the near-surface composition has been systematically determined following numerous sputtering and annealing cycles at different temperatures (see Fig.1(a)). The measurements have been taken to be near-surface sensitive ( $0^{\circ}$ take-off angle) with a sampling depth 


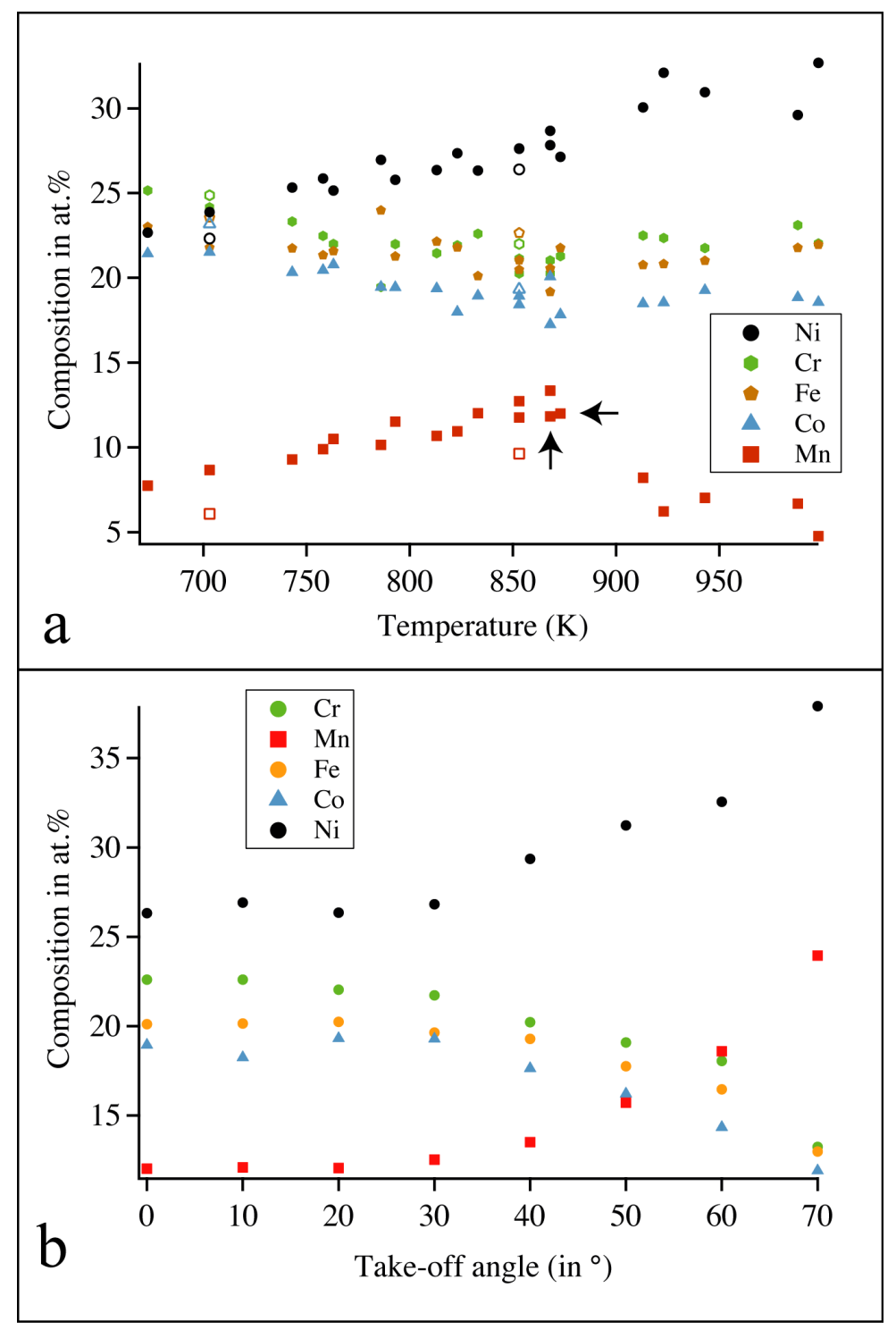

Figure 1: a) Evolution of the atomic composition (in at.\%) of the five elements as a function of the annealing temperature (take-off angle set to $0^{\circ}$ ). The annealing duration differs among the preparations (between 1 to 4 hours). The open symbols indicate that prior to annealing to $703 \mathrm{~K}$ and $853 \mathrm{~K}$, the sample has been heated to $948 \mathrm{~K}$ and $1000 \mathrm{~K}$ respectively. b) Variation of the elemental composition as a function of the photoelectron take-off angle after annealing the sample to $833 \mathrm{~K}$. As the XPS measurements get more surface sensitive $\left(70^{\circ}\right.$ take-off angle), the $\mathrm{Ni}$ and $\mathrm{Mn}$ contents are increasing which is interpreted as a surface segregation of the two elements at the HEA surface. The errors are estimated at $\pm 10 \%$ on the composition and $\pm 20 \mathrm{~K}$ for the temperature across the sample.

of $6.42 \mathrm{~nm}\left(3 \lambda^{15}\right)$. Considering the measurements uncertainties, the individual concentrations of Cr, Fe and Co remain almost constant and around 20 at.\% for the whole temperature 
range explored in this study. However, Fig.1(a) reveals a completely different trend for Mn and Ni. The $\mathrm{Ni}$ and $\mathrm{Mn}$ contents are linearly increasing at the same rate as the annealing temperature for each preparation is increased. This behaviour is observed for the $\mathrm{Mn}$ composition up to $873 \mathrm{~K}$, temperature above which a sudden reduction in the Mn content is identified. This sharp $\mathrm{Mn}$ decrease is associated to its desorption from the surface as opposed to a bulk diffusion. This is demonstrated by the detection of Mn using XPS on a Si(111) surface which has been closely placed above the HEA sample annealed to $933 \mathrm{~K}$.

To evaluate the extent of this surface and near-surface chemical inhomogeneity, angleresolved XPS measurements have been collected after annealing the sample to $833 \mathrm{~K}$. Figure 1(b) confirms unambiguously the surface segregation of $\mathrm{Ni}$ and $\mathrm{Mn}$ at the FeCrMnNiCo sample. When the sampling depth is reduced to $2.2 \mathrm{~nm}$ ( $70^{\circ}$ photoelectron take-off angle), the surface composition is measured at $\mathrm{Fe}_{13.0} \mathrm{Co}_{11.9} \mathrm{Cr}_{13.2} \mathrm{Mn}_{24.0} \mathrm{Ni}_{37.9}$. The Mn content has tripled compared to its nominal stoichiometry and more importantly the slope above $30^{\circ}$ take-off angle is once more identical for $\mathrm{Ni}$ and $\mathrm{Mn}\left(\mathrm{Ni}_{a t . \%}-\mathrm{Mn}_{a t . \%}=\right.$ constant $)$.

Aside from the temperature, the sample preparation conditions can also differ by varying the annealing duration. As illustrated in Fig.1(a), the latter seems to have little effect on the surface stoichiometry, at least within the duration range selected. Indeed, the horizontal and vertical black arrows point to two similar compositions measured after annealing the sample for $1 \mathrm{~h} 25 \mathrm{~min}$ and 4 hours respectively. The lack of duration effect has been repeatedly observed for other annealing temperatures below $873 \mathrm{~K}$.

Another important phenomenon is the sample annealing history which can impact the surface composition. Once the sample has been annealed above $873 \mathrm{~K}$ for more than one hour, there is a clear decrease in the Mn content (see Fig.1(a)). The next preparation cycle, even if carried out below this critical temperature, will result systematically to a lower $\mathrm{Mn}$ surface concentration than expected. This is represented in Fig.1(a) by the two empty square symbols at $853 \mathrm{~K}$ and $703 \mathrm{~K}$, which depart from the almost linear Mn increase (full squares). The annealing conditions used in the preceding preparation cycle are 2 hours at $1000 \mathrm{~K}$ 
and $1 \mathrm{~h} 20 \mathrm{~min}$ at $949 \mathrm{~K}$ respectively. It was shown that several preparation cycles are then necessary to recover the expected surface composition.

As for the XPS measurements, the overlapping of several Auger lines prohibits an accurate determination of the surface chemical composition by Auger electron spectroscopy (AES) in point mode. Instead, the spatial distribution of a given element has been monitored via SAM. This techniques is complementary to XPS which averages over a rectangular millimeter-sized area. Across the (110) and (320) oriented grains, a homogeneous spatial chemical composition has been observed. If demixing or formation of binaries occur at the near-surface, the domain size must be below the highest lateral resolution used during our measurements, i.e. below $20 \mathrm{~nm}$. Randomly taken AES measurements (in point mode) are also quantitatively similar on both orientations except at grain boundaries where variation among Auger line heights are identified. This chemical deviation is better illustrated by Fig.2(b,c) which presents SAM images acquired for Mn LMM and Fe LMM spectra over an area shown in the associated SEM image (Fig.2(a)). The most striking features are a clear enrichment of Mn and a depletion of Fe at the grain boundary, reflected as higher and lower contrast in Fig.2(b,c). Although much less pronounced, a slight increase and decrease are observed also for $\mathrm{Cr}$ and Co respectively at the grain interface (see Fig.S4).

With no Ni segregation measured at grain boundary (bulk (see Fig.5(b)) and surface (see Fig.4S)), the Mn and Ni co-segregation in an equal amount evidenced by angle-resolved XPS should be related to a surface rather than a grain boundary phenomenon. Moreover, considering the estimated area probed by XPS (see Fig.1S), the grain boundary will have a relatively small impact on the overall surface composition, the (110) grain contributing the most to the photoemission measurements. However, we cannot exclude a minute offset of the overall Mn composition from grain boundary surface contribution. 


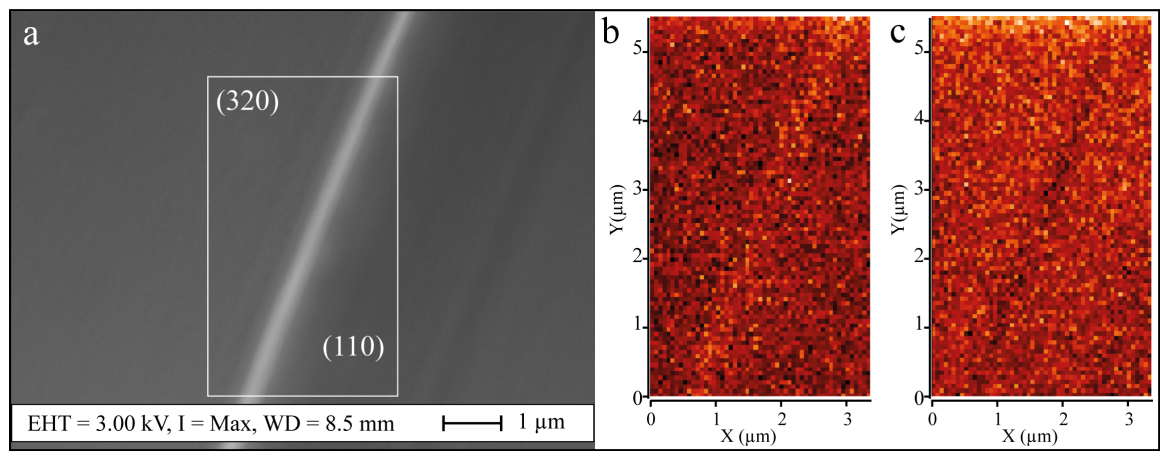

Figure 2: a) SEM image showing the grain boundary between (110) and (320) oriented grains. The white rectangle corresponds to the region for the SAM characterisation shown in (b, c). The SAM images (point size of $63 \mathrm{~nm}$ ) displayed as (Peak-Background)/(Peak+Background) mode reveal the spatial distribution of (b) Mn and (c) Fe elements in the vicinity of the grain boundary. The Mn LMM (582 eV) and Fe LMM (701 eV) spectra have been used for the measurements.

\section{$3.2(110)$ oriented surface}

We now turn to the structural investigation of individual grains by LEED and STM measurements, starting with the (110) surface. Regardless of the annealing temperature used, the LEED patterns exhibit parallel streaks at low energy (Fig.3(a)), diffuse diffraction spots originating from facets and a distinctive $(1 \times 1)$ pattern at $64 \mathrm{eV}$ (Fig.3(b)). The reciprocal lattice vectors are perpendicular to each other with the ratio $b^{*} / a^{*}$ equal to $\sqrt{2}$ as expected for the HEA (110) surface. From the streaks spacing and orientation, local $(2 \times 1)$ reconstructions are expected along the [001] direction and structural disorder should be present in the $[-110]$ directions.

STM measurements have been carried out across the (110) oriented grain for the different annealing temperatures tested (see Fig.1(a)). The results presented hereafter have been obtained after annealing the sample to $810 \mathrm{~K}$ for $1 \mathrm{~h} 20 \mathrm{~min}$. The structure presented in Fig.3(c) should be considered as the most reproducible one among the diverse local structures identified during the surface characterisation. The STM images reveal a rough and highly anisotropic surface morphology. Higher magnification STM images (see Fig.3(d)) show that the structure can be described as atomically flat rows of relative uniform width running 
parallel to the [001] direction. From the height histogram in Fig.3(e), a step height of about $1.20 \AA$ is measured between consecutive rows, in agreement with the interlayer spacing expected for FeCrMnNiCo(110) surface $(1.27 \AA)$.
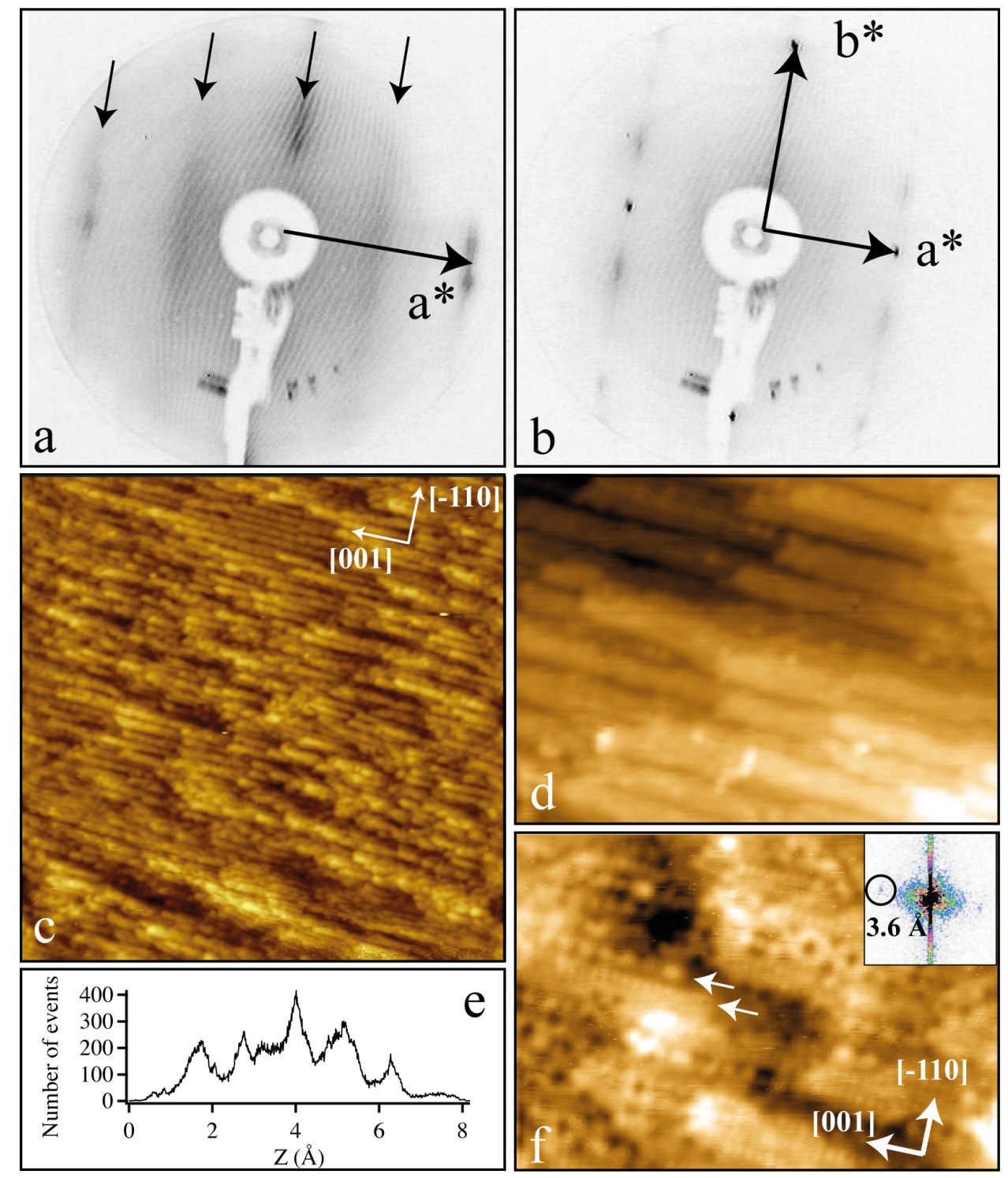

Figure 3: LEED patterns recorded at (a) $30 \mathrm{eV}$ and (b) $64 \mathrm{eV}$ on the (110) grain. c) $1000 \times 1000 \AA^{2}$ STM image revealing an anisotropic surface topography. d) The surface structure resembles rows propagating along the [001] direction $\left(300 \times 220 \AA^{2}\right.$ STM image). e) Step height histogram calculated from the STM image shown in d. f) Atomically resolved STM images $\left(150 \times 105 \AA^{2}\right)$ revealing local reconstruction and a high degree of disorder along the [-110] direction. Inset: Fast Fourier transform calculated from f). The expected (110) surface structure for FeCrMnNiCo is presented in Fig.S5. 
The atomically resolved STM image in Fig.3(f) confirms the LEED analysis. Two types of nanometer-long lines (marked by arrows) can be easily distinguished running parallel to the [001] direction. They are built either by $3.6 \AA$ or by $7.2 \AA$ periodically spaced motifs. The smallest periodicity confirmed by the Fast Fourier transform (FFT) calculations (inset in Fig.3(f)) corresponds to the lattice parameter of the FeCrMnNiCo unit cell. Indeed, the FeCrMnNiCo crystallizes in a face centered cubic structure with $\mathrm{a}=3.596 \AA .^{10}$ The $7.2 \AA$ periodicity should be regarded as a local surface reconstruction. The atomic arrangements along the [-110] direction are much more disordered. It leads to various structural motifs (donut-like shape for instance) and several interatomic spacing, all reflected as streaks in the LEED pattern.

\section{$3.3(320)$ oriented surface}

From the quality of the $(1 \times 1)$ LEED pattern (low background and sharper diffraction spots) shown in Fig.4(a), the FeCrMnNiCo(320) surface is expected to be much more ordered than the (110) surface. The angle $\left(74 \pm 1^{\circ}\right)$ and unit mesh parameters $\left(b^{*} / a^{*} \simeq 1.9\right)$ measured on the LEED pattern are in excellent agreement with those expected for an unreconstructed (320) surface. Contrary to the (110) surface, none of the diffraction spots originate from other crystallographic directions.

As seen in Fig.4(b), the surface structure presents a terrace and step morphology with the majority of step edges aligned along the [001] direction. Consecutive terraces are separated by two main step heights equal to $1.0 \AA$ and $0.5 \AA$ and measured from the height histogram shown in Fig.4(b). These values are consistent with the step heights expected for a (320) oriented surface calculated from $\mathrm{d}_{320}(=3.596 \AA / \sqrt{13})$ and $\mathrm{d}_{320} / 2$ respectively. The structure on each terrace (Fig.4(c)) can be described as parallel rows running along the [001] direction.

Fast Fourier transform calculations of the STM images reveal a row spacing equal to $6.5 \AA$ (see inset in Fig.4(c)). The row structure could not be atomically resolved. However at this stage, dark patches along the rows have been tentatively assigned to point defects and/or 


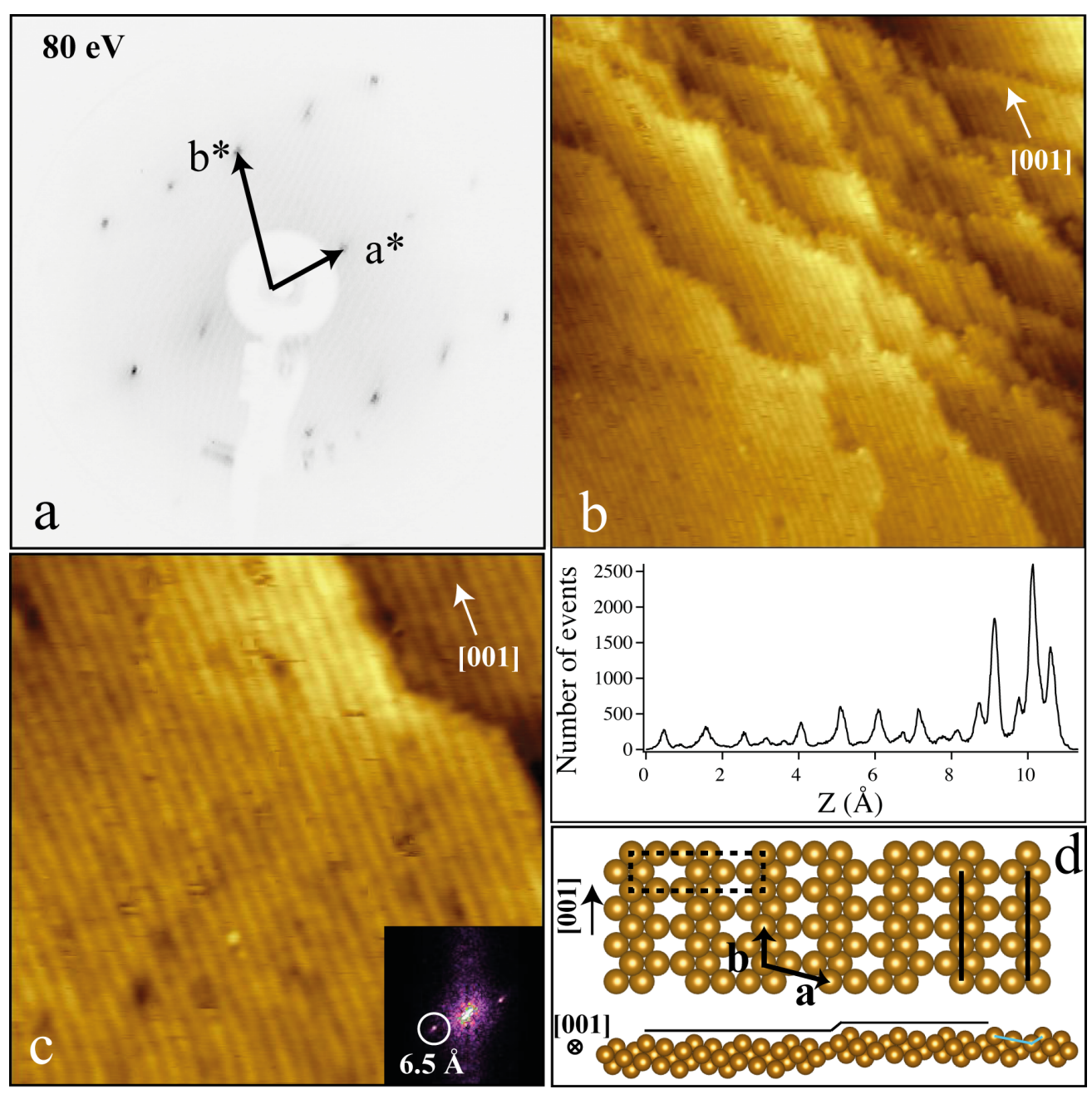

Figure 4: a) LEED pattern recorded on the (320) grain. b) $373 \times 373 \AA^{2}$ STM image showing the terrace steps aligned along the [001] direction. Inset: Step height histogram calculated from the above STM image. c) High resolution STM image $\left(191 \times 191 \AA^{2}\right)$ revealing the surface structure on terraces. Inset: corresponding calculated Fast Fourier transform. d) Top and side views of a fcc (320) surface structure model. The primitive $(6.73 \AA \times 3.60 \AA$; $\left.\alpha=105.5^{\circ}\right)$ and centered $(12.97 \AA \times 3.60 \AA)$ unit cells are indicated. The two lines running parallel to the [001] direction and theoretically spaced by $6.48 \AA$ represent the lines resolved in STM images. The facets (blue line) and the step height (black line) are indicated on the side view of the (320) model.

chemical inhomogeneity. The top and side views of the (320) surface model are shown in Fig.4(d). The surface unit mesh parameters are equal to $\mathrm{a}=6.73 \AA, \mathrm{b}=3.60 \AA$ and $\alpha=$ $105.5^{\circ}$. A larger centered rectangular unit cell ( $\mathrm{a}=12.97 \AA, \mathrm{b}=3.60 \AA$ ) is also indicated on the model. The side view of the (320) surface model represents terraces separated by 
a $1.0 \AA$ step height. The (320) surface is built by two (110) and one (100) facets, i.e. corresponding to a $11.31^{\circ}$ miscut from a (110) surface. This succession of facets leads to an inherent corrugation of the surface which is manifested by the row structure observed using STM. The theoretical spacing of $6.48 \AA$ (experimentally $6.5 \AA$ ) is marked on the model in Fig.4(d) by two parallel black lines. The row structures are shifted perpendicularly to the [001] direction from consecutive terraces with a value depending on the step height. For 1.0 $\AA$ and $0.5 \AA$ step height, the lateral shifts across the step edge are equal to $1.50 \AA$ and 2.49 $\AA$ respectively, which is verified experimentally.

Following the characterization under UHV conditions, the micrometer scale topography and the bulk chemical composition of the (320) and (110) oriented grains have been analysed using SEM and EDS techniques. The SEM measurements reveal a topography for the (110) oriented grain exhibiting parallel striations of micrometer length scale and nanometer width (see Fig.5(a)). The SEM images obtained on the (320) oriented grain are featureless at the observed micrometer scale (see Fig.5(b)). These micrometer observations are reminiscent of the features identified at the nanometer scale by STM, i.e. an anisotropic topography resembling to row-like features on the (110) surface and a terrace and step (i.e. flat) morphology on the (320) surface.
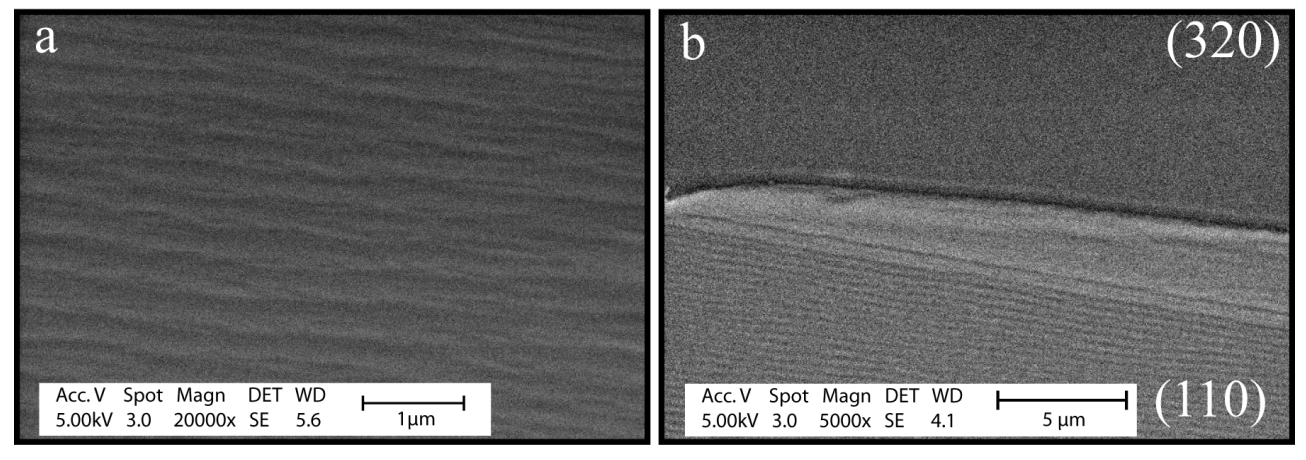

Figure 5: SEM images recorded on (a) (110) grain and (b) at the grain boundary between both single grains.

To determine the HEA chemical composition and potential spatial inhomogeneities, a series of EDS measurements have been performed across the grains and at grain boundaries. 
An identical composition of $\mathrm{Fe}_{23.8} \mathrm{Co}_{22.8} \mathrm{Cr}_{22.3} \mathrm{Mn}_{12.0} \mathrm{Ni}_{19.1}( \pm 0.1$ at.\%) has been found for both grains. However, line profile analysis across the grain boundary shown in Fig.5(b) indicate a clear depletion of Mn content. The morphology of the (110) grain close to the grain boundary appears less streaky and correlate with the reduced Mn concentration measured. Averaging over several single point measurements along the boundary gives a composition of $\mathrm{Fe}_{24.9} \mathrm{Co}_{23.9} \mathrm{Cr}_{22.9} \mathrm{Mn}_{8.7} \mathrm{Ni}_{19.6}$. The compositional discrepancy with the XPS value is attributed to the accuracy associated with the photoemission technique and different probing depth.

\section{Discussion and Conclusion}

Of the two surfaces, our structural analysis demonstrates a greater ordering of the (320) surface consisting of two (110) and one (100) facets. Hence, this surface which is also the most reliable one to prepare, offers a higher degree of intrinsic defects (steps) than the theoretical $\mathrm{FeCrMnNiCo(110)} \mathrm{surface.} \mathrm{As} \mathrm{opposed} \mathrm{to} \mathrm{the} \mathrm{expected} \mathrm{atomic} \mathrm{flat} \mathrm{terraces,} \mathrm{the}$ (110) surface reveals a row-like structure generating a high density of defects (steps, kinks, voids ...) and facets. Further works will be required on other low index FeCrMnNiCo surfaces to understand the role of surface defects and disorder on the surface stability.

The XPS and EDS results confirm the non-equiatomic concentration of the HEA studied here where a Mn depletion is clearly observed (bulk and surface). The chemical analysis of the FeCrMnNiCo surface reveals that preferential sputtering can be disregarded and it also sets the upper annealing temperature to $873 \mathrm{~K}$ under UHV conditions. Above this limit, Mn desorption occurs while Ni concentration at the surface increases steadily up to the highest sample temperature applied in this study $(1000 \mathrm{~K})$, hence departing from the initial quinary HEA concept. The vapor pressure of Mn is about 4 to 5 orders of magnitude higher than for the other elements $\left(1 \times 10^{-9}\right.$ bar at $\left.873 \mathrm{~K}\right)$ which could explain its relatively low desorption temperature. A weak Mn bonding in the bulk could also contribute to this low temperature 
desorption. However, a weak bonding is often reflected by preferential sputtering which is apparently not the case here. ${ }^{16}$

Once the sample has been annealed above $873 \mathrm{~K}$, several preparation cycles are necessary to recover the expected Mn concentration within the surface region, i.e. the sample surface carries a memory effect. This implies that the elemental desorption leads to a Mn depletion in the sub-surface region. The rate of Mn atoms diffusing to the surface does not compensate for the rate of desorption above $873 \mathrm{~K}$. Thus, knowing the sample history is key here to understand the variation of the surface composition. However, it is possible to return to the original surface state under moderate annealing conditions (see Fig.1(a)).

Surface segregation as the one observed here is very common in solid solutions and much less manifested in intermetallics or ordered alloys. ${ }^{17,18}$ The chemical segregation is mainly driven by the atomic radii difference between the elements, the elemental surface energy and the alloy tendency towards chemical ordering or demixing which depends on the sign and magnitude of interatomic interactions. ${ }^{17,19}$ Here, the atomic radii of the five elements present in the FeCrMnNiCo sample are similar. Based on the HEA concept, the formation of intermetallic compounds or phase segregation are a priori not favored (at least at high temperature). It is then likely that the elemental surface energy could be a dominant factor in the surface segregation. Here, Mn exhibits a surface energy of $1.54 \mathrm{~J} / \mathrm{m}^{2}$ which is much lower than the surface energies of the other four elements ${ }^{20}$ ranging between $2.30-2.55 \mathrm{~J} / \mathrm{m}^{2}$. This could explain the segregation of Mn observed at the FeCrMnNiCo surface upon increasing the sample annealing temperature. This is also the driving force proposed to explain the Mn segregation reported recently at the $\mathrm{FeCrMnNiCo}(001)$ topmost surface layer. ${ }^{21,22}$ Moreover, the diffusion rate of $\mathrm{Mn}$ atoms in $\mathrm{FeCrMnNiCo}$ is also the greatest among the elements at high temperature. ${ }^{23}$ This could facilitate the thermally activated Mn enrichment over the other elements at the sample surface (see Fig.1).

Figure 1 demonstrates clearly that Mn segregation is accompanied by Ni surface enrichment in equal proportion, i.e. a co-segregation phenomenon. Ni has one of the slowest 
diffusion rate within the $\mathrm{FeCrMnNiCo}^{23}$ and a comparable surface energy as $\mathrm{Fe}$, Co and $\mathrm{Cr}$, raising the question, what is the mechanism leading to its surface segregation?

The FeCrMnNiCo system is considered as a non-ideal HEA. As such the interatomic forces between every pairs of atoms are not equal and the enthalpy of mixing is non-zero (as opposed for instance to the ideal HoDyYGdTb HEA ${ }^{24}$ ). Thus, it appears that the Mn-Ni pair enthalpy of mixing is the most negative one $\left(\Delta \mathrm{H}^{m i x}{ }_{M n N i}=-8 \mathrm{~kJ} / \mathrm{mol}\right)$ among all pairs which could favor clustering of the two elements (ordering). This effect has been reported by Otto et al. ${ }^{25}$ after annealing at $773 \mathrm{~K}$ for 500 days for homogenised single phase FeCrMnNiCo ingots. At heterogeneous sites (grain boundaries, inclusions, intergranular voids), the HEA solid solution decomposes to form a tetragonal $\mathrm{Ni}$ and $\mathrm{Mn}$ rich phase, a Cr-rich $\mathrm{BCC}$ phase and an ordered Fe and Co-rich cubic phase. For a similar HEA sample but subjected to high pressure torsion, Schuh et al. ${ }^{26}$ have reported the formation of $\mathrm{MnNi}$ precipitates along with Cr-rich phase after just 5 min annealing to $723 \mathrm{~K}$. Upon increasing the annealing time, their respective volume fractions are increasing and a FeCo phase appears. ${ }^{26}$ For the Cantor alloy aged at $723 \mathrm{~K}$, Li et al. have also demonstrated co-segregation of $\mathrm{Ni}$ and $\mathrm{Mn}$ at highangle grain boundaries, the main driving force being the highly negative energy of mixing of the two elements. ${ }^{27}$ These studies show the propensity to develop MnNi phases at grain boundaries and defect sites at relatively low annealing temperature. Under the conditions used in our study, there is no evidence for Ni-Mn precipitates at grain boundaries in the bulk or at the surface. In fact, we observed by EDS a bulk Mn depletion at the grain boundary (see Fig.5(b)). From a surface perspective, the adsorption of Mn adatoms on Ni(100) leads also to the growth of an ordered $\mathrm{Mn}_{50} \mathrm{Ni}_{50}$ film which adopts a tetragonal structure. ${ }^{28}$ Within the FeCrMnNiCo system, the coherent growth of other Mn-Ni cubic phases, having a lattice parameter close to the HEA one, could have been expected.

The annealing temperature range reported in the previous works ${ }^{25-27}$ is comparable to the one used in the present study, although the annealing duration and sample treatments differ. Therefore, a decomposition of the single-phase solid solution into (inter)-metallic 

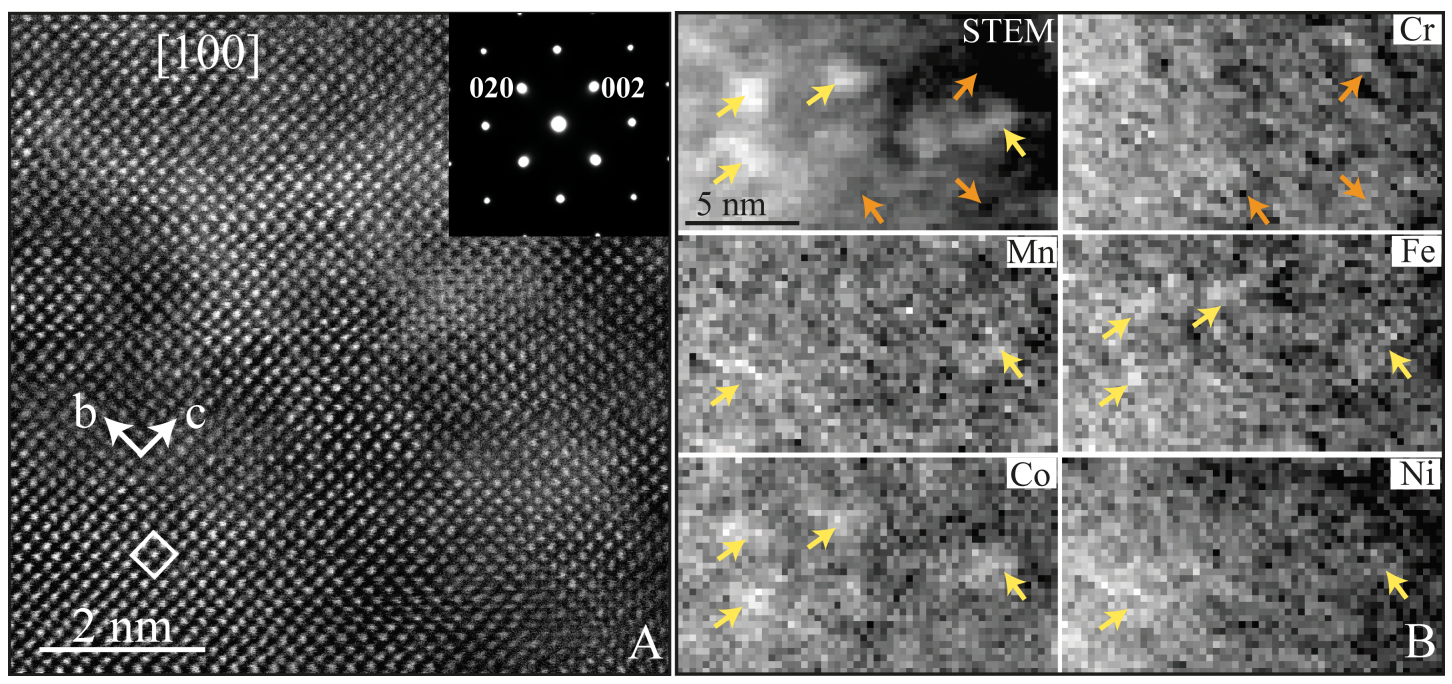

Figure 6: (a) Atomically resolved HAADF-STEM image with the corresponding SAED pattern acquired from the (110) oriented grain along the [100] zone axis. (b) HAADF-STEM image (top left) and corresponding elemental EELS maps revealing small local variations in composition. The yellow and orange arrows are eye-guides to highlight correlative areas of increased and decreased elemental concentration signal respectively. The FIB lamella thickness is measured around $50 \mathrm{~nm}$.

phases could occur. Thus, the bulk and subsurface regions of (110) and (320) grains have been characterised by TEM. Structural and chemical analysis have been performed using selected-area electron-diffraction (SAED) pattern analysis, high-angle annular dark field (HAADF)-STEM, EELS and EDS (not shown here) techniques on different thin FIB FeCrMnNiCo lamellae cut perpendicularly to the two studied surfaces. These cross-sectional observations reveal a chemically homogeneous bulk and subsurface region at the nanoscale after repeated annealing cycles. An atomically resolved HAADF-STEM image is presented in Fig.6(a) exhibiting very homogeneous contrast with few local heterogeneities. The elemental EELS maps collected in parallel (see Fig.6(b)) indicate small compositional variations at the atomic scale. Thus, the Co signal (one of the most prominent one) perfectly correlates with the increased STEM signal. Furthermore, there is a faint but detectable positive correlation of Fe with Co. Additional correlations between Mn and Ni have been highlighted in Fig.6(b) where also increased Co and Fe signals are present. Regarding the Cr element, the corresponding signal is increased in areas with a lower intensity on STEM image and 
on $\mathrm{Mn}, \mathrm{Fe}, \mathrm{Co}$ and Ni elemental maps. SAED pattern (shown as the inset in Fig.6(a)) was acquired from the same specimen region where HAADF-STEM image was obtained with the selected-area diameter of approximately $150 \mathrm{~nm}$. The corresponding pattern analysis reveals a single-phase system, the fcc crystal structure with the unit cell of $3.6 \AA$. These results demonstrate that there is no phase decomposition but instead local chemical variations (2-4 at.\% as measured on EELS and EDS plot profiles) compatible with the HEA concept. As explained previously, there is also no evidence for the formation of nanoscale precipitates within the topmost surface layers (see Fig.2(b,c)).

To conclude, we propose that Mn surface segregation is mainly driven by its low surface energy. Within the surface region, this elemental enrichment alters considerably the local chemical composition and distribution compared to the nominal HEA bulk stoichiometry. In fine, this chemical surface variation will impact the HEA phase stability. Because of their mutual attraction and tendency to form binary phases, Mn surface enrichment will promote Ni surface segregation. Thus, Mn segregation should be regarded as the driving force to facilitate diffusion of $\mathrm{Ni}$ to the surface, leading to co-segregation of the two elements in equal proportion. A consequence of Mn high vapor pressure is its desorption from the HEA surface above $873 \mathrm{~K}$. Above this temperature, the outgoing flux of Mn atoms leads to a Ni-rich topmost surface. While the largest, the Mn diffusion rate does not compensate the rate of desorption. Having highlighted a morphology difference between the (320) and (110) surfaces, these results set also the upper temperature limit for the use of FeCrMnNiCo coating under medium to high vacuum conditions and for aerospace applications.

\section{Declaration of Competing Interests}

The authors declare that they have no known competing financial interests or personal relationships that could have appeared to influence the work reported in this paper. 


\section{Acknowledgement}

This work is a result of cooperation within the European C-MAC and within the FrenchSlovene collaboration established under Push-Pull Alloys and Complex Compounds (PACS 2$)$ Joint Open Laboratory. JL acknowledges the INC CNRS and the Université de Lorraine for financially supporting the IRP PACS 2 . We acknowledge the funding of our research activities by the Slovenian Research Agency through the program P2-0084. MF received funding from the German Research Foundation (DFG) under grant number FE 571/4-1 within the priority program SPP2006.

\section{References}

(1) J.W. Yeh, S.K. Chen, S.J. Lin, J.Y. Gan, T.S. Chin, T.T. Shun, C.H. Tsau, and S.Y. Chang, Nanostructured High-Entropy Alloys with Multiple Principal Elements: Novel Alloy Design Concepts and Outcomes, Adv. Eng. Mater. 6, 299 (2004).

(2) M. Feuerbacher, M. Heidelmann, and C. Thomas, Hexagonal High-entropy Alloys, Mater. Res. Lett. 3, 1 (2015).

(3) W. Steurer, Single-phase High-entropy Alloys A Critical Update, Mater. Charact. 162, 110179 (2020).

(4) P. Kozelj, S. Vrtnik, A. Jelen, S. Jazbec, Z. Jaglicic, S. Maiti, M. Feuerbacher, W. Steurer, and J. Dolinšek, Discovery of a Superconducting High-Entropy Alloy, Phys. Rev. Lett. 113, 107001 (2014).

(5) P. Kozelj, S. Vrtnik, A. Jelen, M. Krnel, D. Gacnik, G. Drazic, A. Meden, M. Wencka, D. Jezersek, J. Leskovec, S. Maiti, W. Steurer, and J. Dolinšek, Discovery of a FeCoNiPdCu High-Entropy Alloy with Excellent Magnetic Softness, Adv. Eng. Mater. 21, 1801055 (2019). 
(6) O.N. Senkov, S.V. Senkova, C. Woodward, and D.B. Miracle, Low-density, Refractory Multi-principal Element Alloys of the Cr-Nb-Ti-V-Zr System: Microstructure and Phase Analysis, Acta Mater. 61, 1545 (2013).

(7) O.N. Senkov, D.B. Miracle, K.J. Chaput, and J.-P. Couzinié, Development and Exploration of Refractory High Entropy AlloysA Review, J. Mater. Res. 33, 3092 (2018).

(8) B. Cantor, I.T.H. Chang, P. Knight, and A.J.B. Vincent, Microstructural Development in Equiatomic Multicomponent Alloys, Mater. Sci. Eng. A 375, 213 (2004).

(9) B. Gludovatz, A. Hohenwarter, D. Catoor, E.H. Chang, E.P. George, and R.O. Ritchie, A fracture-Resistant High-entropy Alloy for Cryogenic Applications, Science 345, 1153 (2014).

(10) Q. Ye, K. Feng, Z. Li, F. Lu, R. Li, J. Huang, and Y. Wu, Microstructure and Corrosion Properties of CrMnFeCoNi High Entropy Alloy Coating, Appl. Surf. Sci. 396, 1420 (2017).

(11) S. Yin, W. Li, B. Song, X. Yan, M. Kuang, Y. Xu, K. Wen, and R. Lupoi, Deposition of FeCoNiCrMn High Entropy Alloy (HEA) Coating via Cold Spraying, J. Mater. Sci. Technol. 35, 1003 (2019).

(12) J. Li, Y. Huang, X. Meng, and Y. Xie, A Review on High Entropy Alloys Coatings: Fabrication Processes and Property Assessment, Adv. Eng. Mater. 21, 1900343 (2019).

(13) Y. Yao, Z. Huang, P. Xie, S.D. Lacey, R.J. Jacob, H. Xie, F. Chen, A. Nie, T. Pu, M. Rehwoldt, D. Yu, M.R. Zachariah, C. Wang, R. Shahbazian-Yassar, J. Li, and L. Hu, Carbothermal Shock Synthesis of High-entropy-alloy Nanoparticles, Science 359, 1489 (2018).

(14) Weighing in more Mn could compensate this loss, approach used for arc-melted FeCoNiCrMn alloy by F. Otto, Y. Yang, H. Bei, and E.P. George, Relative effects of 
enthalpy and entropy on the phase stability of equiatomic high-entropy alloys, Acta Materialia 61, 2628 (2013).

(15) L. Wang, D. Mercier, S. Zanna, A. Seyeux, M. Laurent-Brocq, L. Perrière, I. Guillot, and P. Marcus, Study of the Surface Oxides and Corrosion Behaviour of an Equiatomic CoCrFeMnNi High Entropy Alloy by XPS and ToF-SIMS, Corrosion Science 167, 108507 (2020).

(16) C.D. Yuen, B. Ünal, D. Jing, and P.A. Thiel, Weak Bonding of Zn in an Al-based Approximant based on Surface Measurements, Phil. Mag. 91, 2879 (2011).

(17) U. Bardi, The Atomic Structure of Alloy Surfaces and Surface Alloys, Rep. Prog. Phys. 57, 939 (1994)

(18) J. Ledieu, É. Gaudry, and V. Fournée, Surfaces of Al-based Complex Metallic Alloys: Atomic Structure, Thin Film Growth and Reactivity, Sci. Technol. Adv. Mater. 15, $034802(2014)$

(19) G. Tréglia, B. Legrand, F. Ducastelle, A. Saúl, C. Gallis, I. Meunier, C. Mottet, and A. Senhaji, Alloy Surfaces: Segregation, Reconstruction and Phase Transitions, Comput. Mater. Sci. 15, 196 (1999).

(20) L. Vitos, A.V. Ruban, H.L. Skriver, and J. Kollár, The Surface Energy of Metals, Surf. Sci. 411, 186 (1998).

(21) P. Wynblatt and D. Chatain, Modeling Grain Boundary and Surface Segregation in Multicomponent High-Entropy Alloys, Phys. Rev. Mater. 3, 054004 (2019).

(22) D. Chatain and P. Wynblatt, Surface Segregation in Multicomponent High Entropy Alloys: Atomistic Simulations versus a Multilayer Analytical Model, Comput. Mater. Sci. 187, 110101 (2021). 
(23) K.-Y. Tsai, M.-H. Tsai, and J.-W. Yeh, Sluggish Diffusion in Co-Cr-Fe-Mn-Ni Highentropy Alloys, Acta Materialia 61, 4887 (2013).

(24) J. Lužnik, P. Koželj, S. Vrtnik, A. Jelen, Z. Jagličić, A. Meden, M. Feuerbacher, and J. Dolinšek, Complex Magnetism of Ho-Dy-Y-Gd-Tb Hexagonal High-entropy Alloy, Phys. Rev. B 92, 224201 (2015).

(25) F. Otto, A. Dlouhý, K.G. Pradeep, M. Kuběnová, D. Raabe, G. Eggeler, and E.P. George, Decomposition of the Single-phase High-entropy Alloy CrMnFeCoNi after Prolonged Anneals at Intermediate Temperatures, Acta Materialia 112, 40 (2016).

(26) B. Schuh, F. Mendez-Martin, B. Völker, E.P. George, H. Clemens, R. Pippan, and A. Hohenwarter, Mechanical Properties, Microstructure and Thermal Stability of a Nanocrystalline CoCrFeMnNi High-entropy Alloy after Severe Plastic Deformation, Acta Materialia 96, 258 (2015).

(27) L. Li, R.D. Kamachali, Z. Li, and Z. Zhang, Grain Boundary Energy Effect on Grain Boundary Segregation in an Equiatomic High-Entropy Alloy, Phys. Rev. Mater. 4, $053603(2020)$.

(28) M. Wuttig and C.C. Knight, LEED Structure Determination of Tetragonal MnNi films on Ni(100), Phys. Rev. B 48, 12130 (1993). 